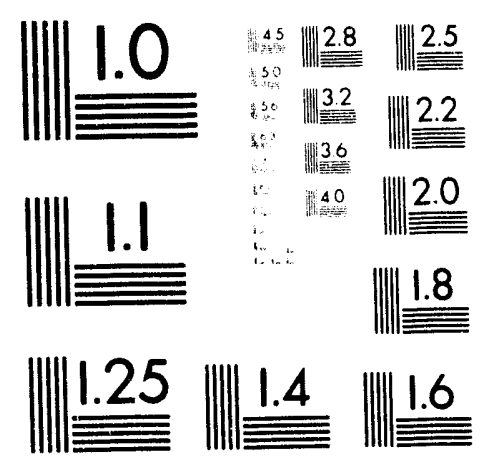



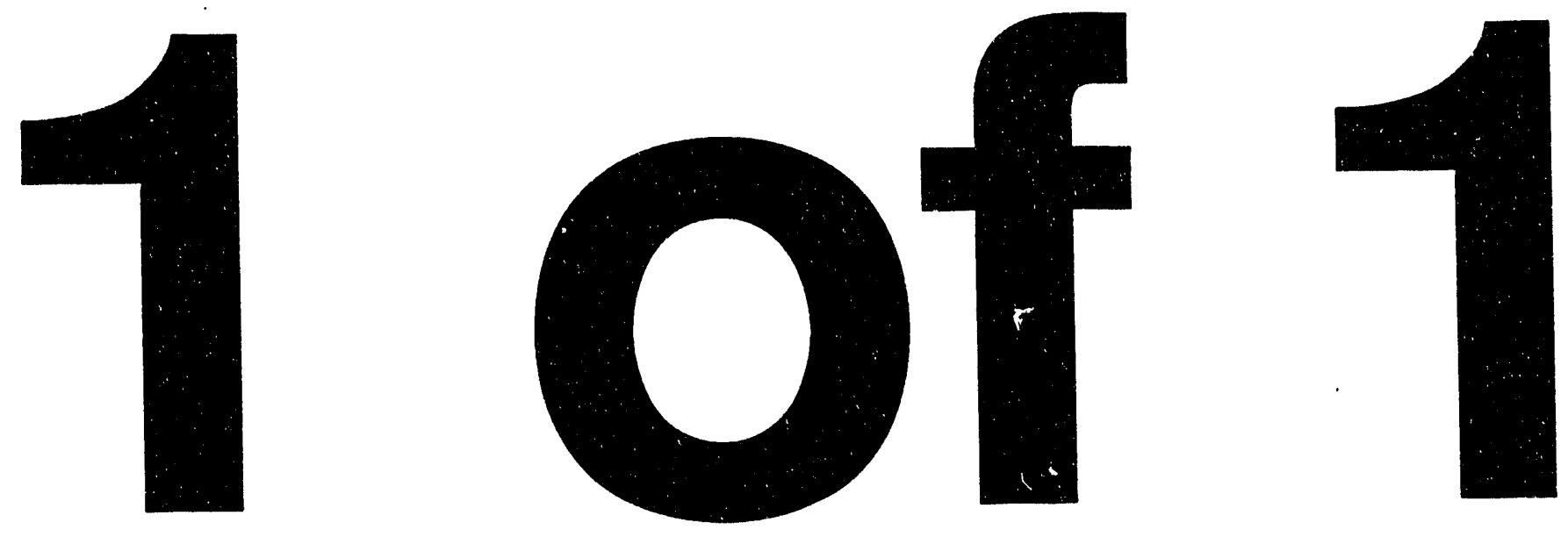
$\frac{1}{20.93} 98 \Omega$

UCRL-ID- 115116

\title{
Performance Evaluation of Video on Ethernet
}

\author{
Mike Pihlman
}

Renee Farrell

This is an informal report intended primarily for intemal or limited extemal distribution. The opinions and conslusions stated are those of the author and may or may not be those of the Laboratory.

Work performed under the auspices of the U.S. Department of Energy by the Lawrence Livermore National Laboratory under Contract W-7405-Eng-48.

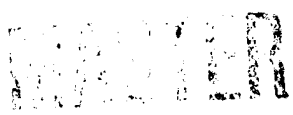




\section{DISCLAIMER}

This document was prepared as an account of work sponsored by an agency of the United States Government. Neither the United States Government nor the University of California nor any of their employees, makes any warranty, express or implied, or assumes any legal liability or responsibility for the accuracy, completeness, or usefulness of any information, apparatus, product, or process disclosed, or represents that its use would not infringe privately owned rights. Reference herein to any specific commercial products, process, or service by trade name, trademark, manufacturer, or otherwise, does not necessarily constitute or imply its endorsement, recommendation, or favoring by the United States Government or the University of California. The views and opinions of authors expressed herein do not necessarily state or reflect those of the United States Government or the University of California, and shall not be used for advertising or product endorsement purposes.

This report has been reproduced directly from the best available copy.

A vailable to $D O E$ and $D O E$ contractors from the Office of Scientific and Technical Information P.O. Box 62, Oak Ridge, TN 37831

Prices a vailable from (615) 576.8401, FTS 626-8401

A vailable to the public from the

National Technical Information Service

US. Department of Commerce

5285 Port Royal Rd.,

Springfield, VA 22161 


\section{Performance Evaluation of Video on Ethernet}

Small Business Initiative Project \#93001-

by

Mike Pihlman

Renee Farrell

Lawrence Livermore National Laboratory (LLNL)

Distributed Computing Support Program (DCSP)

\section{Executive Summary}

This paper summarizes the results of a Small Business Initiative Project (\#93001) that the Lawrence Livermore National Laboratory (LLNL) recently completed for Compression Labs, Inc. (CLI).

The purpose of this project was to ztermine the feasibility of using an ethernet local area network (LAN) to support videoconferencing connections between CAMEO Macintosh desktop videoconferencing systems. The specific goals were to:

1. Ensure that CAMEO video could be transported-without protocol modification-via existing ethernet networks, and would do so without "bringing-down" the network.

2. Measure the effect of CAMEO video connections on ethernet traffic.

3. Evaluate qualitatively how generated ethernet traffic effects the CAMEO video.

4. Evaluate qualitatively how multiple CAMEO connections work between two routered ethernet networks via a backbone.

High quality CAMEO video can be transported on an ethernet network and between routered networks, via a backbone. The number of simultaneous video connections possible on an ethernet segment would probably be less than 45 , since each connection uses $2.2 \%$ of the network and errors increase rapidly as video connections are made. However, the actual number of simultaneous video connections possible will depend upon your network implementation and the amount of "normal" traffic present. The remainder of this report discusses the effect of CAMEO video on our networks.

Please note that although CAMEO video has been tested and shown to work on the networks at Lawrence Livermore National Laboratory, we cannot ensure that you will achieve the same results on your network.

\section{Brief Technical Overview}

Ethernet uses a Carrier Sensed Multiple Access/Collision Detect (CSMA/CD) media access scheme that allows multiple devices to "democratically" share a common transmission path. If a device has information to transmit and the path is busy, that device will backoff a random amount of time before attempting to send its information again. Once the path is free the information can be exchanged. This access method is very efficient for time independent bursty traffic, but, video and audio information is constant, and time sensitive so the delays caused by waiting for the path can cause problems which can be seen as video breakup and heard as audio delays and crackle. If traffic on the ethernet increases (as it quickly would with added video sources), the path becomes busier, more re-tries are attempted, and more collisions (requiring re-transmissions) occur causing the delays to increase. Eventually the ethernet network could saturate ${ }^{i}$ causing all information flow to cease.

\footnotetext{
${ }^{1}$ It is generally believed that the ethernet will saturate at an offered load of approximately $37 \%$. Boggs, Mogul, and Kent (reference 1) show that that number may be misleading and state that a properly designed, and used, ethernet network is "capable of carrying its nominal bandwidth".
} 
Proper design of an ethernet network may allow you to transport video, in reasonable numbers, without unduly effecting normal traffic. Reference 1 gives the following guidelines for proper ethernet implementation:

-Don't install long cables: to cover a large area, break up the cable with bridges or routers, not repeaters.

-Don't put too many devices on one cable: use routers to break the network into communities of interest, trading higher delay for inter-community traffic for better intra-community response time and throughput.

- Implement the protocol correctly: proper collision detection and binary-exponential backoft in software is essential to good performance.

- Use largest possible packet size: this keeps the packet count down, reducing the likelihood of collision and not incidentally reducing overheads internal to devices.

-Don't mix serious real-time and serious bulk data applications: it is not possible to simultaneously guarantee the lowest delay and the highest throughput (although for moderate requirements both kinds of applications coexist well).

New neiwork hardware and protocols are emerging that will permit efficient video and audio transport on LANs. Some companies claim to be able to transport video at very high bit rates (over $1 \mathrm{Mbps}$ ) on existing ethernet with minor protocol modifications. Others are introducing "fast" ethernet networks and "switched" ethernet networks that can provide full ethernet bandwidth to each user or that increase the common path bandwidth to $100 \mathrm{Mbps}$. Asynchronous Transfer Mode (ATM) is on the horizon promising to provide anywhere between $1.544 \mathrm{Mbps}$ to1 Gbps to the desktop.

\section{Test Procedure}

To avoid possible problems, and to understand the effects of video transport on the ethernet, we initially performed the video tests on an isolated "test" network (figure 1). When we were convinced that the CAMEO would not saturate the ethernet we connected the test network to the operational network and completed the tests both within that segment and between segments via the backbone.

All of the tests listed below were repeated 10 times and averages taken. The tests in step 1 were taken on various days and times for 30 minutes at a time. During testing, the CAMEO was operating at $50 \mathrm{kbps}$ using the Apple ADSP tool on a mixture Macintoshes using either Asante or Apple ethernet cards.

We performed the following tests:

1. Measured utilization, "runt" frame errors, CRC/alignment errors, total frame errors, missed/lost frames, total bytes, average frame size, and file transfer times for:

+ Isolated network with $0,1,2$, and 3 point-to-point video connections +Operational network with $0,1,2$, and 3 point-to-point video connections (intrazone)

2. Measured file transfer times between two Appleshare servers (413 kbyte file) on:

+ Isolated network with 0,1 , and 2 point-to-point video connections +lsolated network with $20 \%$ generated traffic and 1 point-to point connection +lsolated network with $50 \%$ generated traffic and 1 point-to point connection

3. Qualitative tests were done to observe CAMEO operation:

+with $80 \%$ and $95 \%$ simulated traffic

+with 2 CAMEO connections between routered segments via the backbone 


\section{Test Configuration}

Figure 1 shows the test configuration we used. The test network consists of a 12 port 10BaseT HP hub connecting 7 CAMEO systems, a printer (not shown), and a Network General "Sniffer" LAN Analyzer. The test network is connected to the main network by a pair of Tutankhamon 10BaseT to single twisted pair wire network extenders (XL600+), via an approximately 200 foot length twisted pair wire.

We isolated the test network by disconnecting the link to the main network at the test networks hub. Once we were convinced that video traffic would not bring down the ethernet, we reconnected the networks and performed the tests indicated on the operational network. We tested inter-zone connectivity by supplying CAMEOs to personnel in a building located approximately $3 / 4$ mile from the test network. The networks at both sites are connected to a backbone ethernet via CISCO routers.

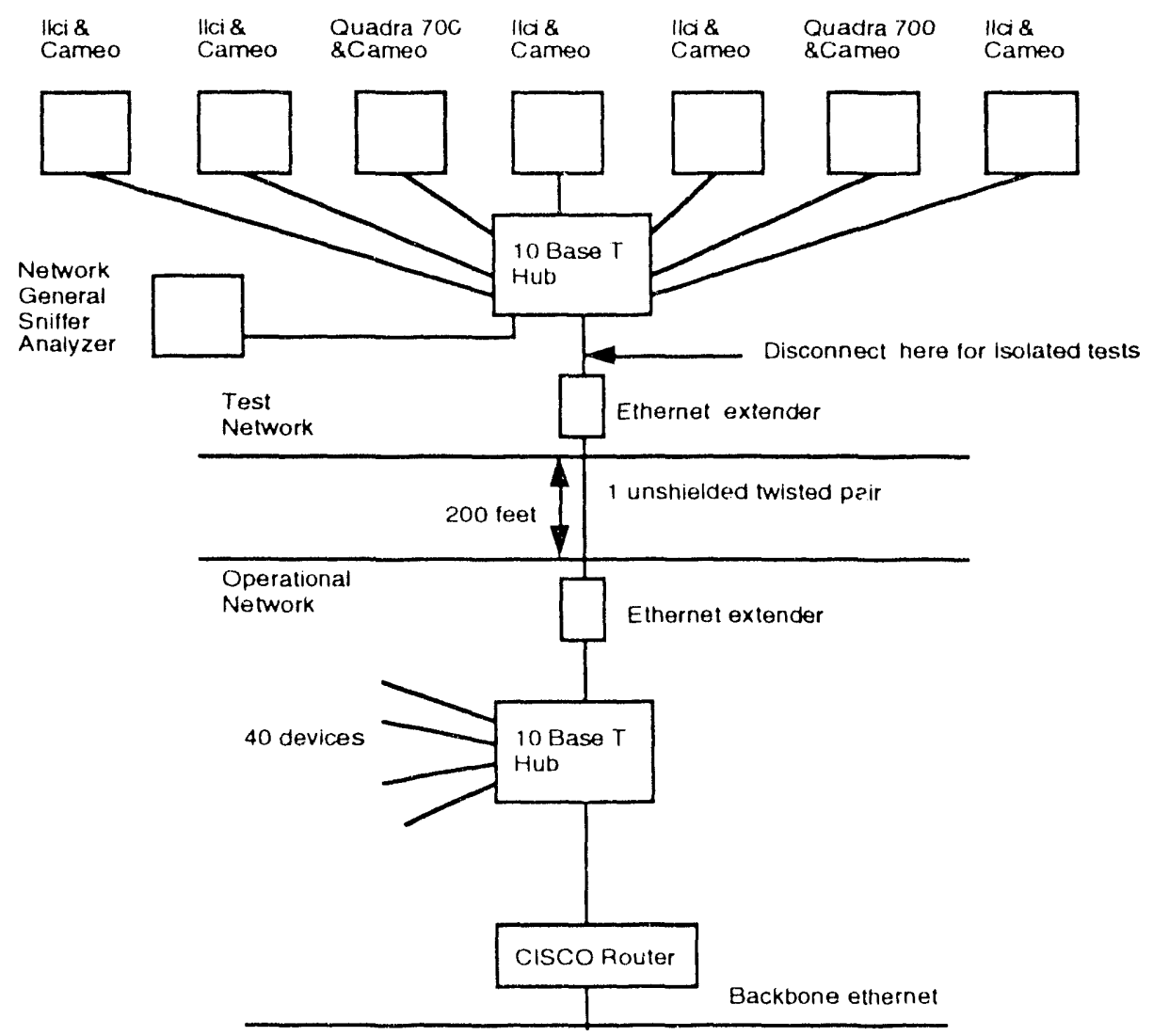

Figure 1-Test Configuration and Simplified Depiction of Network Connection to LabNet 


\section{Test Results}

The following tables summarize the results of the study.

\begin{tabular}{|c|c|c|c|c|}
\hline Test & No video & 1 video & 2 video & 3 video \\
\hline Utilization & $0.3 \%$ & 2.57 & 4.95 & 6.97 \\
\hline $\begin{array}{l}\text { "Runt" frame } \\
\text { errors }\end{array}$ & 0 & 0 & 0 & 0 \\
\hline $\begin{array}{l}\text { CRC/alignment } \\
\text { errors }\end{array}$ & 0 & 0 & 0 & 0 \\
\hline Total frame errors & 0 & 0 & 0 & 0 \\
\hline $\begin{array}{l}\text { Missed/lost } \\
\text { frames }\end{array}$ & 0 & 0 & 0 & 0 \\
\hline Total Frames & 17,866 & 420.199 & 767.020 & $1,124,285$ \\
\hline Total bytes & $6,508,569$ & $52,414,624$ & $93,102,976$ & $129,965,539$ \\
\hline $\begin{array}{l}\text { Average frame } \\
\text { (packet) size } \\
\text { (bytes) }\end{array}$ & 364 & 124 & 12.1 & 115 \\
\hline & & & & \\
\hline
\end{tabular}

Table 1-Isolated Network Test Results

\begin{tabular}{|l|l|l|l|l|}
\hline Test & No Traffic & 1 video & 2 video & 3 video \\
\hline Utilization & $1.397 \%$ & $4.048 \%$ & $6.153 \%$ & $8.106 \%$ \\
\hline $\begin{array}{l}\text { "Runt" frame } \\
\text { errors }\end{array}$ & 13.1 & 117.4 & 287 & 477.33 \\
\hline $\begin{array}{l}\text { CRC/alignment } \\
\text { errors }\end{array}$ & 0 & 4 & 1 & 1.33 \\
\hline Total frame errors & 13.1 & 117.8 & 288 & 478.66 \\
\hline $\begin{array}{l}\text { Missed/lost } \\
\text { frames }\end{array}$ & 0 & 0 & 0 & 0 \\
\hline Total Frames & 143,991 & 525,870 & 897,295 & $1,301,802$ \\
\hline Total bytes & $28,287,517$ & $79,276,178$ & $117,925,136$ & $156,852,142$ \\
\hline $\begin{array}{l}\text { Average frame } \\
\text { (packet) size } \\
\text { (bytes) }\end{array}$ & 193 & 149.6 & 131 & 119.66 \\
\hline & & & & \\
\hline
\end{tabular}

Table 2-Operational Network Test Results

\section{Utilization}

Utilization is a measure of how busy a network is. Table 2 shows that the operational networks normal traffic usage was a very low $1.397 \%$ of the network. Each video connection adds to that by approximately $2.2 \%$. Graph 1 shows how the video data quickly dominates the lightly used network. With this rate of increase, we estimate that the ethernet network would probably saturate before 45 video connections could be made. 


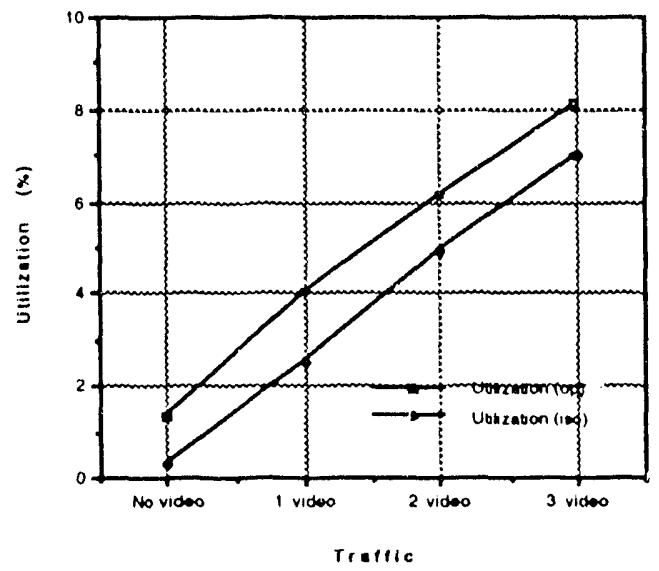

Graph 1-Utilization

\section{"Runt" Frame Errors}

Runt errors are caused by collisions on the common path. The isolated network had no errors of this type. The frame errors on the operational network increase dramatically as video connections are added. Although this appears frightening, the number of errors compared to the total number of bytes and frames (packets) transmitted is quite low $(.03 \%)$. It's probable (but we couldn't test it) that errors may increase non-linearly at higher traffic loads due to the increased probability of collisions.

"Runt" Frame Errors

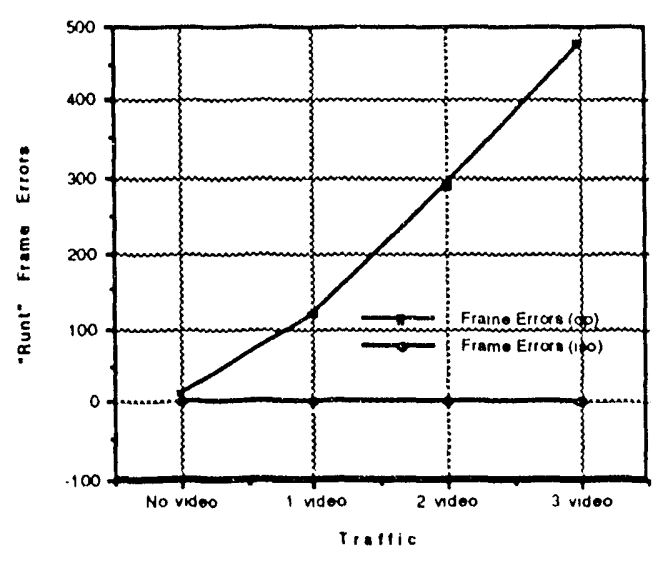

Graph 2-"Runt" Frame Errors

\section{CRC/Alignment Errors}

CRC/Alignment errors are errors that have occurred in transmission and were detected by checking the Cyclical Redundancy Checksum (CRC) code at the end of the packets. These values show a tendency to increase as video connections increase, but, they are so small as to be negligible. However, as in the case of runt errors, this value may increase sharply as the utilization increases. 


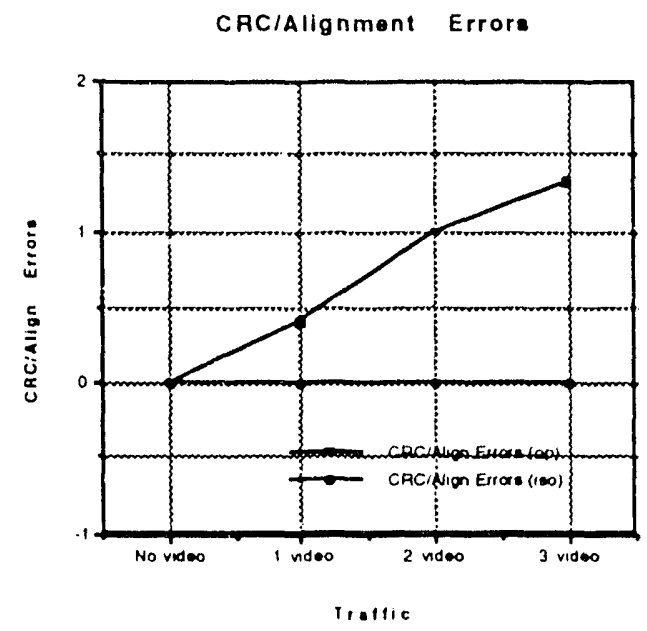

Graph 3-CRC/Alignment Errors

\section{Total Frames}

The total number of frames transmitted increases as video connections are added. Each video source was calculated to generate approximately 200 packets per second. The packet length of the CAMEO was 115 bytes. Reference 1 states that ethernet would achieve better utilization of the network if larger packets were used.

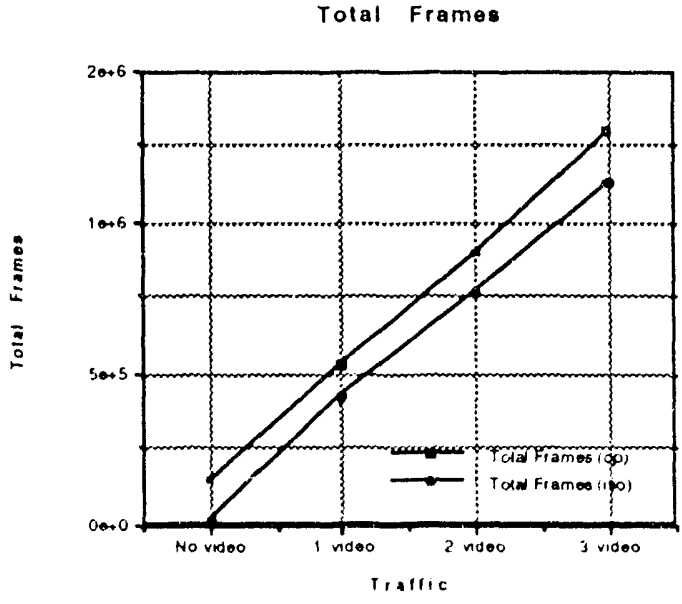

Graph 4-Total Frames

\section{File Tiansfer Times}

The file transfer times were conducted on the isolated to avoid misreadings due to traftic on the operational network and to allow us to generate traffic with the Sniffer. It is interesting to note that even at $50 \%$ generated traffic and 1 video connection, the file transfer times, although slower than with no video and normal traffic, was still not trad. The video at $50 \%$ generated traffic appeared equivalent to the video at $1.397 \%$ traffic. 


\begin{tabular}{|l|l|l|l|l|}
\hline Measurement & No Video & $\begin{array}{l}20 \% \text { generated } \\
\text { traffic }\end{array}$ & $\begin{array}{l}20 \% \text { generated } \\
\text { traffic and 1 } \\
\text { video }\end{array}$ & $\begin{array}{l}50 \% \text { generated } \\
\text { traffic and 1 } \\
\text { video }\end{array}$ \\
\hline mean & & & & \\
\hline Std Dev & 5.997 & 7.283 & 7.473 & 9.681 \\
\hline
\end{tabular}

Table 3-File Transfer Times Isolated Network

\section{Conclusions}

CAMEO video can be transported, without apparent loss of quality, on an ethernet network and between routered networks, via a backbone. If the number of video connections are limited the CAMEO video does not severely effect the network. We found that networks with $50 \%, 80 \%$, and $95 \%$, simulated traffic can support video connections (quality is degraded at $80 \%$ and $95 \%$ ). The number of simultaneous video calls on a network depends upon several factors such as the throughput rates of routers/bridges, the bit rate of any wide area links the video is traversing, and the traffic loading/distribution of the network. Taking all this into account, an existing ethernet LAN will probably saturate before 45 simultaneous video connections can be made.

Figure 2 shows an example of how a network may support 20 simultaneous CAMEO connections. Three ethernet networks are connected to a backbone via routers or bridges. In the top network, 10 CAMEO connections are underway using $22 \%$ of the network. None of this traffic is present on the backbone. The two bottom networks also have 10 connections established using $22 \%$ of both bottom networks and $22 \%$ of the backbone (assuming the backbone is also $10 \mathrm{Mbps}$ ethernet). If the backbone is $100 \mathrm{Mbps}$, then we only use $2.2 \%$ of the backbone, thus allowing more inter-segment calls to be made.

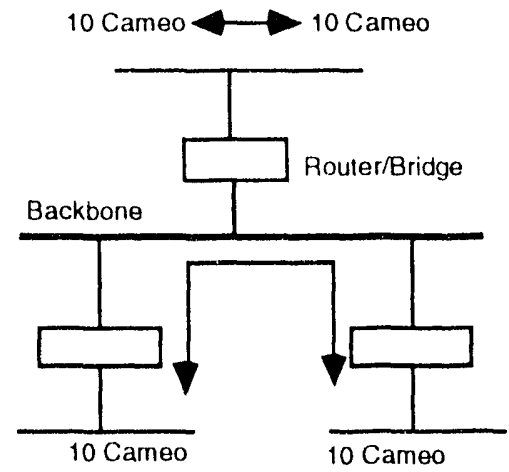

Figure 2-Example of a Possible CAMEO Connection Scenario

Reference 2 has an interesting chart that shows the maximum possible packet transfer rates for $56 \mathrm{kbps}$, T1 (1.544 Mbps), and ethernet routered connections. We have interpolated the approximate maximum transfer rates for a 115 byte packet.

\begin{tabular}{|l|l|l|l|}
\hline Packet Size & $56 \mathrm{kbps}$ & $1.544 \mathrm{Mbps}$ & Ethernet \\
\hline 64 bytes & & & \\
\hline 1,518 bytes & $120 \mathrm{pps}$ & $3,300 \mathrm{pps}$ & $14,800 \mathrm{pps}$ \\
\hline & $5 \mathrm{pps}$ & $139 \mathrm{pps}$ & $812 \mathrm{pps}$ \\
\hline 115 bytes (CAMEO) & $116 \mathrm{pps}$ & & \\
\hline
\end{tabular}


Since each video connection produces approximately $200 \mathrm{pps}$, a $56 \mathrm{kbps}$ routered link may not be useful for wide area video connections. A T1 connection will saturate at approximately 15 video connections, and the ethernet will saturate at approximately 71 video connections. From the results of our experiment, we found that ethernet will saturate at approximately 45 video connections.

\section{Recommendations for Further Work/Study}

We make the following recommendations for follow-on study and/or future work. Some of these could be used as Small Business Initiative Projects with other companies developing products, systems or networks.

1. Measure the effect of various video bit rates on the ethernet network.

2. Measure the effect of various video packet sizes on the network.

3. Evaluate video traffic on networks with a heavier load.

4. Evaluate the effect of MBONE connections effects ethernet.

5. Evaluate performance of video on "switched" and/or "fast"ethernet.

6. Evaluate performance of video on ATM networks.

7. Evaluate modified protocols that give video and audio priority on exisling ethernet networks.

\section{References}

1. "Measured Capacity of an Ethernet: Myths and Reality", David Boggs, Jeffery Mogul, and Christopher Kent, WRL Research Report 88/4, September 1988.

2. "Ethernet Bridges and Routers: Faster than Fast Enough", Scott Bradner, Data CommunicationsVol 21, No.3, February 1992, pages 58-69

\section{Acknowledgments}

We would like to thank the following people for helping us with this project. David Sauerhaft, Mike Stauffer and their design team at CLI for their "above and beyond" assistance in making this project happen. Dieter Fuss and Peggy Poggio for their organizational support in the Distributed Computing Support Program (DCSP). Tina Darmohray and Dave Wiltzius for their very helpful technical discussions of ethernet, protocols, bridges, and routers. 

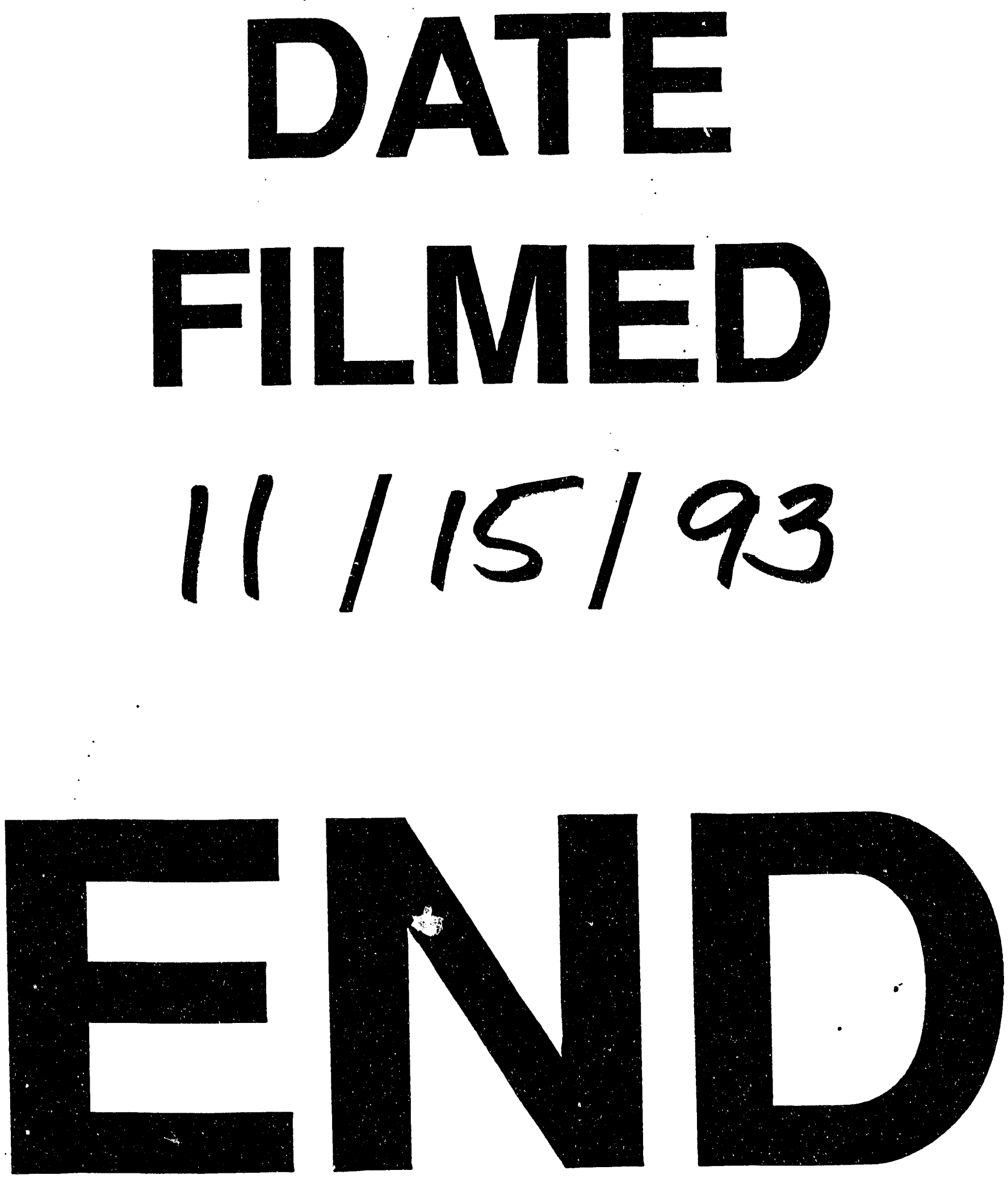